University of Nebraska - Lincoln

DigitalCommons@University of Nebraska - Lincoln

Faculty Publications: Materials Research

Science and Engineering Center

Materials Research Science and Engineering

Center

2007

\title{
Sequential Stretching Lithography
}

\author{
Haojing Lin \\ University of Nebraska - Lincoln \\ Ocelio V. Lima \\ University of Nebraska - Lincoln \\ Li Tan \\ University of Nebraska-Lincoln, Itan4@unl.edu \\ Zheng Li \\ University of Nebraska - Lincoln \\ Jiangyu Li \\ University of Washington, jjli@uw.edu
}

Follow this and additional works at: https://digitalcommons.unl.edu/mrsecfacpubs

Part of the Materials Science and Engineering Commons

Lin, Haojing; Lima, Ocelio V.; Tan, Li; Li, Zheng; and Li, Jiangyu, "Sequential Stretching Lithography" (2007). Faculty Publications: Materials Research Science and Engineering Center. 79.

https://digitalcommons.unl.edu/mrsecfacpubs/79

This Article is brought to you for free and open access by the Materials Research Science and Engineering Center at DigitalCommons@University of Nebraska - Lincoln. It has been accepted for inclusion in Faculty Publications:

Materials Research Science and Engineering Center by an authorized administrator of DigitalCommons@University of Nebraska - Lincoln. 


\title{
Sequential Stretching Lithography
}

Haojing Lin ${ }^{1}$, Ocelio V Lima ${ }^{1}$, Li Tan ${ }^{1}$, Zheng $\mathrm{Li}^{1,2}$, and Jiangyu $\mathrm{Li}^{3}$

${ }^{1}$ Department of Engineering Mechanics and Nebraska Center for Materials and Nanoscience, University of Nebraska, Lincoln, NE, 68588

${ }^{2}$ Department of Mechanics and Engineering Science, Peking University, Beijing, China, People's Republic of

${ }^{3}$ Department of Mechanical Engineering, University of Washington, Seattle, WA, 89195

\begin{abstract}
We developed an embossing/imprinting based nanofabrication technique, dubbed sequential stretching lithography (SSL). In this process, a master pattern is imprinted into an elastomer containing a film of uncured elastomer. The elastomer is cured and then elongated to increase feature density and reduce feature size. Replication of this substrate yields a new master that can be used in further reduction steps. One-dimensional grating features with a pitch size below $200 \mathrm{~nm}$ were fabricated from $750 \mathrm{~nm}$-pitch grating lines. This process gives us a faithful pattern miniaturization in all aspects and, as a result, a much effective control on density and dimension regulation.
\end{abstract}

\section{INTRODUCTION}

Over the last years, Nanoscience and Nanotechnology has seen a significant amount of efforts aimed at producing well-defined nanostructures and they can be divided into so called 'top-down miniaturization' or 'bottom-up synthesis' approaches. Major top-down nanofabrication techniques include extreme UV photolithography (EUV), e-beam lithography (EBL), scanning probe lithography (SPL), focused ion beam lithography (FIB), embossing/imprinting lithography and among others. Extreme UV and X-rays have demonstrated the resolution down to $100 \mathrm{~nm}$, however, optics capable of supporting a robust and cost-effective process still faces significant challenges [1]. Although a resolution as good as a few or tens of nanometers has been achieved in SPL, EBL and FIB respectively, it is impractical for mass production due to their serial-writing and thus, low-throughput nature [2]. On the other hand, embossing/ imprinting has been proven as a low cost, parallel processing method for direct patterning of polymers [3]. In practice, a layer of polymer thin film is cast over or displaced by a mold or stamp to produce copies of desired structural features. Feature sizes measuring 5 nanometers have been reported [4]. Further miniaturization using these methods, though, is not trivial due to the precision requirements in mold making and alignments between steps. One of the challenges, for instance, is to regulate feature density on the parent mold, thus affording tunability in structure dimension and density among copies. This acute need forms the scope of this paper, where a new nanofabrication technique that integrates feature density tunability into the easy-to-process nature of embossing/imprinting is presented.

Many efforts have been invested to afford manufacture flexibility to embossing/imprinting, in which small scale structures with size or shape different from those on the master stamp were demonstrated. Pathways contributing to such flexibility mainly include a fine tuning of master stamp deformation in vertical direction [5], such as a multi-layered master stamp design or a careful control on pressuring the soft stamp. Feature size miniaturization, on 
the other hand, was performed through a designated dewetting or diffusion process on the stamped material [6]. Formidable challenges yet remain to attenuate feature density. Recently, we presented the concept of tuning micro- or nanostructures through in-plane contraction of the substrate [7], which could miniaturize embossed feature dimension aggressively and, at the same time, increase pattern density. However, the contraction nature of the process introduces undesired features too, which include a film thickness dependent miniaturization ratio and instability of soft structures after a drastic pattern width reduction.

Inspired by the cold-drawing techniques developed for metallic compounds to compose micro- or nanoscale rods/wires [8], we designed a new process, dubbed sequential stretching lithography or SSL, which takes the easy-to-process nature of embossing/imprinting to create $750 \mathrm{~nm}$ pitch grating lines on soft substrates and then, utilizes uni-axial stretching along longitudinal direction of grating features to achieve an significant pitch reduction to $200 \mathrm{~nm}$. In this process, a master pattern is imprinted into an elastomer containing a film of uncured elastomer. The elastomer is cured and then elongated to increase feature density and reduce feature size. Replication of this substrate yields a new master that can be used in further reduction steps. Comparing to previously demonstrated contraction approach [7], SSL gives us a faithful pattern miniaturization in all aspects and, as a result, a much effective control on density and dimension regulation. This paper reports both experimental work and tightly coupled simulations.

\section{MATERIALS AND CHARACTERIZATIONS}

Chemicals were purchased from Sigma-Aldrich and used as received unless otherwise stated. The elastic material used is poly (dimethylsiloxane) (PDMS, Sylgard 184®, viscosity of $5000 \mathrm{cSt}$ ) from Dow Corning. The elastic films were prepared by vigorous mixing of a 10:1 ratio of part A $(5.0 \mathrm{~g})$ and part $\mathrm{B}(0.5 \mathrm{~g})$ in a plastic beaker followed by degassing in a vacuum chamber. Cured elastic thin films were then cut into long strip $(4.5 \times 1.0 \mathrm{~cm})$ and used as substrate for soft mold replication. Elastic films are usually stretched $80-100 \%$ elongation. Stretching and relaxing of the elastic films are manually performed by using a home-made setup and the rate of such deformation is estimated to be of $20 \mathrm{~mm} / \mathrm{s}$.

Features created from each step were investigated by atomic force microscope (AFM, Dimension 3100 and Nanoscope IIIa, Veeco). AFM images were acquired in tapping mode (512 lines). A silicon microcantilever with an etched conical tip (radius of curvature of $30 \mathrm{~nm}$ ) was used for the scanning and the short cantilever has a nominal resonant frequency of $300 \mathrm{kHz}$ and a spring constant of $40 \mathrm{~N} / \mathrm{m}$.

\section{RESULTS AND DISCUSSION}

The strategy of SSL in dimension miniaturization and nanostructure formation is shown in Figure 1. Within this SSL process, synthetic polymer capable of large elastic deformation is used as substrate and is coated with a thin layer of non-cured liquid pre-elastomer (Step 1). A patterned rigid mold is then brought into contact with this coated substrate and this whole assembly is baked to cure the pre-elastomer layer underneath the rigid mold (Step 2). Subsequently, the rigid mold is peeled off, which gives rise to an elastic mold $\left(P_{1}\right)$ with negative mold features (Step 3). Up to this point, the procedure is identical to the conventional embossing/imprinting, and the process starts to deviate from conventional embossing/imprinting 
from the next step. Pattern density increase and dimension reduction in $P_{1}$ mold occurs after $P_{1}$ is stretched along longitudinal dimension of the grating lines (Step 4). Further replication into rigid structures $\left(P_{2}\right)$ allows cycling of this SSL process (Steps 5\&6), such that nanostructures with a feature size of $P_{n}=P_{1} \times R^{n}$ can be achieved in principle by repeating the stretching multiple times (i.e., $n$ times), where $\mathrm{R}$ is the miniaturization ratio of each cycle.

The soft membrane used in SSL was formed by spin-coating the poly(dimethylsiloxane) (PDMS) precursor mixture on a rigid mold at $300 \mathrm{rpm}$ for $30 \mathrm{~s}$; and baked at $80^{\circ} \mathrm{C}$ for $2 \mathrm{hrs}$. In order to obtain a uniform pattern on PDMS, it is necessary to treat the rigid mold ( $\mathrm{Si}$ or $\mathrm{SiO}_{2}$, grating lines with a pitch size of $750 \mathrm{~nm}$, a linewidth of $350 \mathrm{~nm}$ and a depth of 100-120 nm) with $\mathrm{O}_{2}$ plasma, followed by a perfluorosilane treatment in toluene $(0.2 \mathrm{M}, 5 \mathrm{~min}$.). This patterned PDMS thin film is firstly fixed on a home-made stretcher and was elongated along the longitudinal direction of grating features. This mechanical stretching will decrease the feature dimension and increase the pattern density. Then epoxy $(0.1 \mathrm{~mL}, 2$ Ton Epoxy® from Devcon CO., non-cured) was applied to the elongated film, and a glass slide $(7.5 \times 2.5 \mathrm{~cm})$ was placed atop the epoxy to ensure a good wetting to the PDMS surface. After the epoxy was cured at $60^{\circ} \mathrm{C}$ for 8 hours, the elongated PDMS was peeled off, completing the initial cycle and giving rise to a miniaturized topography in epoxy $\left(P l^{\prime}\right)$. This new mold is used to replace the rigid mold in Step 1 and a fresh PDMS precursor is cast atop a solid PDMS, followed by embossing/imprinting of fresh PDMS precursor with this new mold. The imprinting is carried out by forming an intimate contact between the mold and PDMS bi-layer, then baking the whole assembly. Repetition of above processes continues cycling of the SSL. Figure 2 shows AFM topographical and cursor plots of all the molds fabricated in each individual cycles of SSL.

In order to evaluate the miniaturization ratio in each individual elongation step, we assume the soft material to be incompressible. Such that, with an elongation in the x-direction in the coordinate shown in Figure 3a-insert, overall volume of the soft membrane is conserved and strains in either $\mathrm{y}$ or $\mathrm{z}$ direction can be expressed using the formula,

$$
\varepsilon_{y}=\varepsilon_{z}=\frac{1}{\sqrt{1+\varepsilon_{x}}}-1
$$

where $\varepsilon$ stands for strain inside the membrane and each subscript represents a strain on that particular direction, for instance, $\varepsilon_{\mathrm{x}}$ is the strain along the direction of elongation. Figure 3a illustrates the non-linear relationship between applied strain in elongation direction (x) and resulted feature change due to strains in y- or z-axis. It is clear that $\varepsilon_{\mathrm{y}}$ or $\varepsilon_{\mathrm{z}}$ increases slightly when $\varepsilon_{\mathrm{x}}$ is made more than $100 \%$. This trend guides our elongation on soft membrane in the range of $80-100 \%$ to ensure a robust and repeatable processing.

Both pitch and depth on fabricated molds are measured, using AFM, at multiple sample points for each surface and the surface on replicated sample is chosen to ensure a good correlation with that on parent mold. The data are then averaged before comparison between mirror surfaces and are indicated in Table 1, where theoretical data on pattern regulation is also shown. Clearly, pitch size in the new mold is greatly reduced after 5-cycle SSL to $197 \mathrm{~nm}$ from that in original mold $(750 \mathrm{~nm})$; and data on pitch dimension decrease in each individual cycles of SSL matched closely with above theoretical expectations (5\% error). The depth reduction in the first cycle of SSL matched with theoretical expectation (Table 1). The experimental data indicate that the depth loss from original mold (pitch of $750 \mathrm{~nm}$ ) to PDMS, or from PDMS to epoxy, is minimal (less than $0.8 \%$ ). However, the depth reduction of more than $15 \%$ in following cycles of SSL is observed, which is much larger than those theoretical expectations (Table 1). We note 


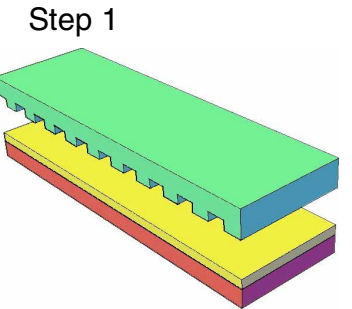

Step 4

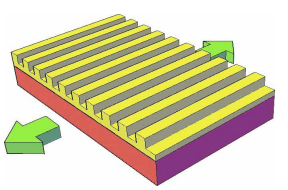

Step 2

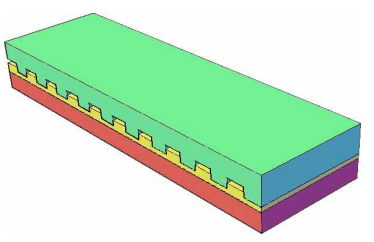

Step 5

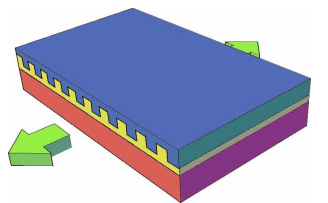

Step 3

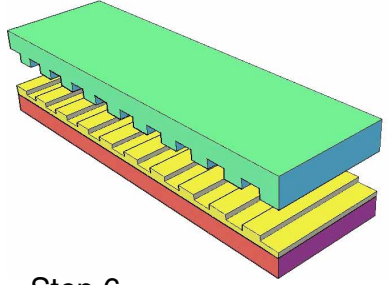

Step 6

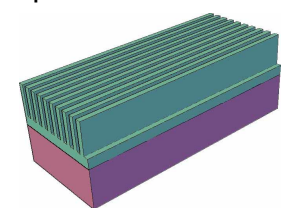

Figure 1. Process flow for Sequential Stretching Lithography (SSL) and they are: (Step 1) coat a pre-elastomer (liquid) over a soft substrate; (Step 2) embossing/imprinting using a rigid mold to displace liquid materials underneath the mold; (Step 3) solidify the liquid precursor after baking and remove the mold; (Step 4) stretch the soft pattern to regulate feature dimension and density; (Step 5) Replicate the topographical features to a hard material; and (Step 6) Repeat steps (1)-(5) until reach the desired dimension or density
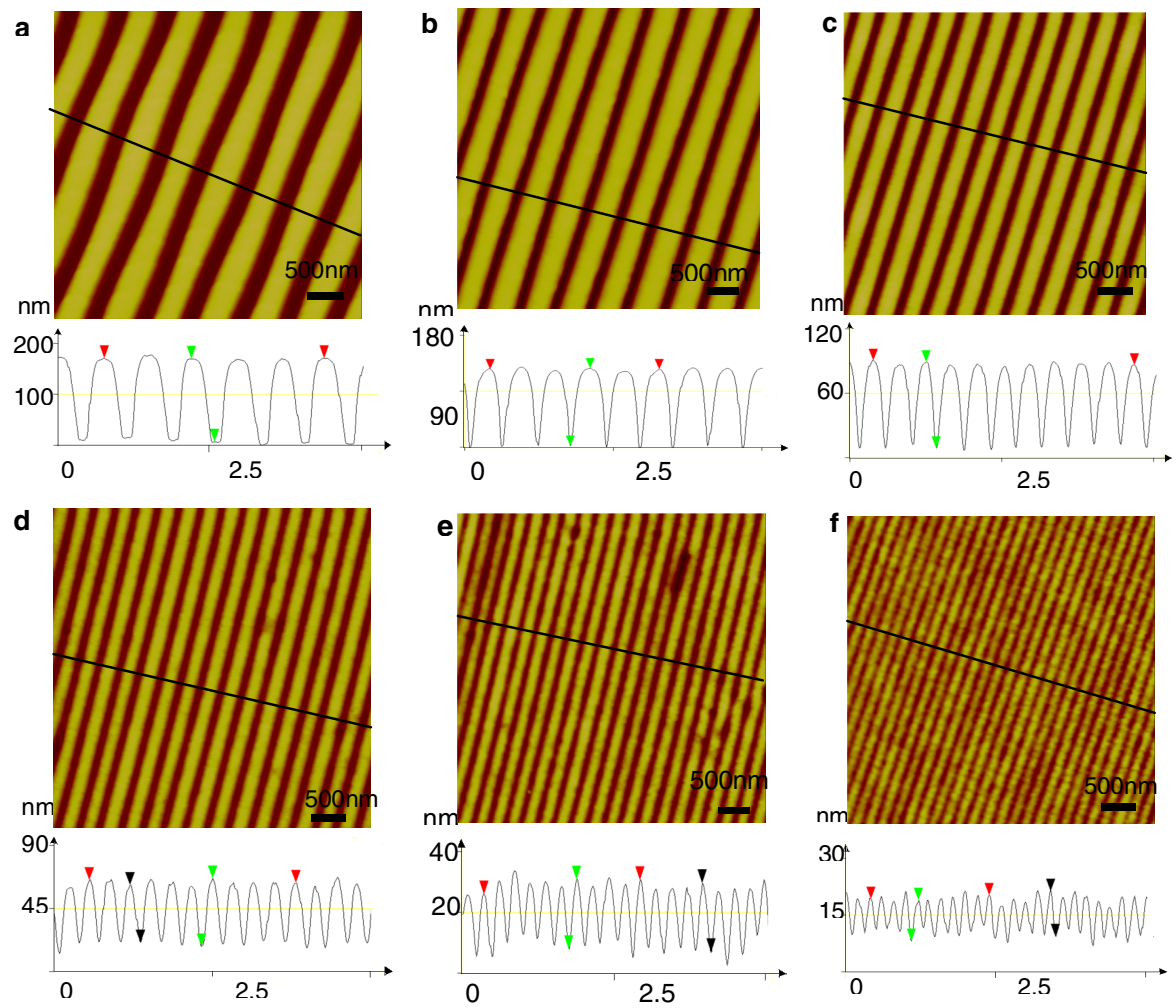

Figure 2. AFM topographical and cursor plots of features atop rigid mold. (a) original mold with a pitch of $750 \mathrm{~nm}$ and (b)-(f) new molds fabricated after individual cycle of SSL, where (f) shows a pitch of $200 \mathrm{~nm}$. 


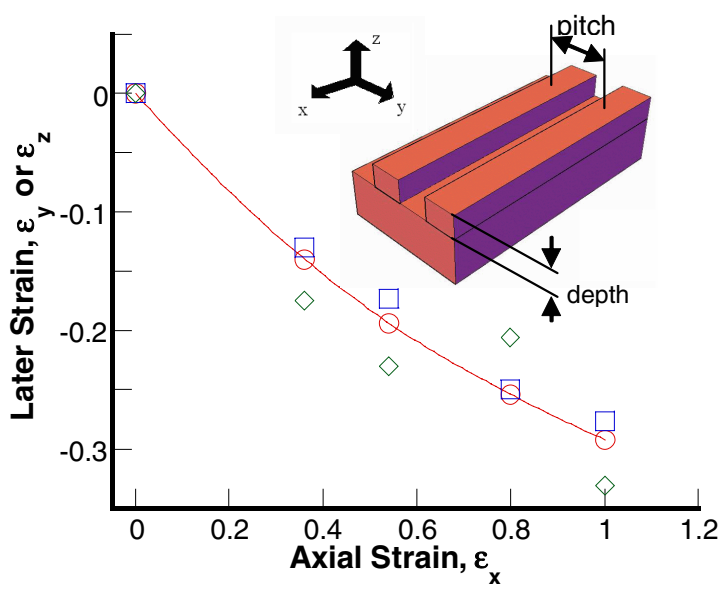

(a)

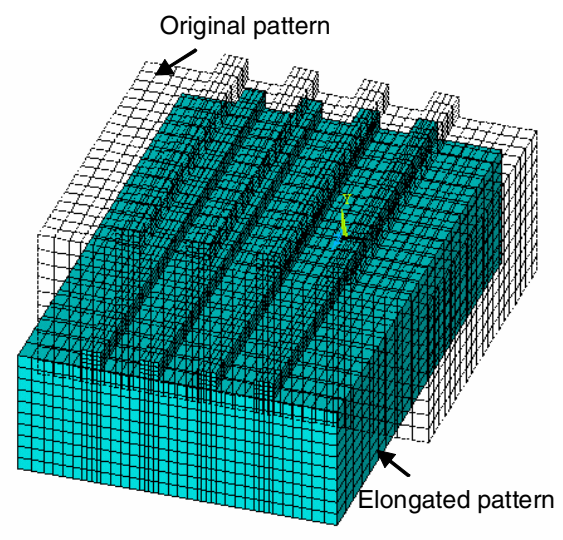

(b)

Figure 3. (a) Feature depth and pitch change due to a lateral strain caused by an axial elongation: (circle) theoretical lateral strain; (square) experimental data on pitch change; and (diamond) experimental data on depth change; and (b) 3-D pattern profile change after an external axial strain, modeled by finite element analysis (ANSYS).

Table 1. Key dimensions of feature pitch and depth after the Sequential Stretching Lithography

\begin{tabular}{lccccc}
\hline & $\mathbf{1}^{\text {st }}$ cycle & $\mathbf{2}^{\text {nd }}$ cycle & $\mathbf{3}^{\text {rd }}$ cycle & $\mathbf{4}^{\text {th }}$ cycle & $\mathbf{5}^{\text {th }}$ cycle \\
\hline Strain & 0.80 & 0.75 & 0.60 & 0.75 & 0.60 \\
\hline Theoretical pitch, nm & 540 & 410 & 324 & 244 & 203 \\
Experimental pitch, nm & 543 & 429 & 328 & 257 & 197 \\
\hline Theoretical depth, $\mathbf{~ m m ~}$ & 120 & 96 & 59 & 37 & 18 \\
Experimental depth, nm & 127 & 80 & 49 & 23 & 12 \\
\hline \hline
\end{tabular}

that there is a likelihood of an incomplete material filling during the process of replicating, for instance, replicating soft PDMS by epoxy or using this epoxy mold to emboss/imprint a new PDMS, respectively. Major reasons for such an incomplete filling are perhaps due to a surface tension difference between the parent mold and the replication material or low diffusion rate for filling materials of large viscosities. The combination of these two reasons becomes more evident at smaller dimensions due to the so-called capillary effects. Particularly, we studied this dependence at a pitch size of $560 \mathrm{~nm}$. When epoxy is used to duplicate the topography on soft PDMS (a pitch of $562 \mathrm{~nm}$ and a depth of $122 \mathrm{~nm}$ ), the copied surface has a depth of $114 \mathrm{~nm}$, indicating a depth loss of 7\%. Moreover, when PDMS was utilized to replicate this epoxy mold, a depth loss of more than $20 \%$ is observed. That is to say, the main depth loss occurred when PDMS was allowed to diffuse into epoxy grooves. We suspect that the major barrier for an easy filling is due to the large viscosity of the PDMS mixture. In order to improve the replication process for a larger depth, we used toluene to dilute PDMS precursor. This diluted PDMS has a weight concentration of $83.3 \%$ and it replicates well the same epoxy mold, giving a depth of 120 $\mathrm{nm}$. Therefore, using solvent to dilute PDMS in the SSL process is a promising way to minimize depth loss.

To reveal pattern profile change due to the SSL process, nonlinear finite element analysis (FEA) is used to simulate the uni-axial stretching, in which 3-D stress state in thin membrane is computed by ANSYS. Since the elastic material is not compressible, a hyperelastic constitutive model (Ogden type) is used to describe the associated deformation. Parameters $\left(\mu_{1}=0.30 \mathrm{MPa}\right.$ 
and $\alpha_{1}=4.92$ ) of the model for PDMS are informed with curve fitting the stress-strain data (not shown). Soft PDMS structure, featured with grating lines of $350 \mathrm{~nm}$ in width and a spacing of $750 \mathrm{~nm}$, is divided with SOLID185 element (3-D 8-node) and illustrated in Figure 3b. Clearly, when an axial strain is applied on soft membrane, features on this soft membrane, i.e., grating lines, are elongated and this elongation leads to a uniform size reduction in patterned features, including both pitch and depth. Another significant merit, comparing to previously established contraction process [7], is the capability to increase pattern density while keep the profile or cross-section of features to be proportional (Figure 3b).

\section{CONCLUSIONS}

This paper demonstrates a new technique, dubbed sequential stretching lithography, which takes the easy-to-process nature of embossing/imprinting to create $750 \mathrm{~nm}$ pitch grating lines on soft substrates and then, utilizes uni-axial stretching along longitudinal direction of grating features to achieve a significant amount of pitch reduction to $200 \mathrm{~nm}$. Comparing to previously demonstrated contraction approach, SSL gives us a faithful pattern miniaturization in all aspects and, as a result, a much effective control on density and dimension regulation. The pitch reduction, as well as depth loss, after each individual SSL cycle is evaluated by both experimental and theoretical data. We found out that capillary effect of the replication process starts to play when pitch size is reduced. This could potentially affect the fabrication resolution of SSL and further experiment to reveal such a limit is under progress.

\section{ACKNOWLEDGMENTS}

The project described was made possible by Nebraska Tobacco Settlement Biomedical Research Development Fund, Charles J. Millard Trust Fund, Nebraska EPSCoR first award and NSF MRSEC Seed grant. One of the authors, Dr. Zheng Li, was partially supported by the exchange program between Peking University and the University of Nebraska-Lincoln.

\section{REFERENCES}

1. J. Jonkers, Plasma Sources Sci. T., 15(2), S8-S16 (2006).

2. G.Y. Liu, S. Xu and Y.L. Qian, Acc. Chem. Res., 33, 457-466 (2000)

3. S. Y. Chou, P.R. Krauss and P.J. Renstrom, J. Vac. Sci. Technol. B, 14, 4129-4133 (1996)

4. M.D. Austin, H.X. Ge, W.Wu, M.T. Li, Z.N. Yu, D. Wasserman, S.A. Lyon, S.Y. Chou, App. Phys. Lett., 84, 5299-5301 (2004)

5. J. Tien, C. M. Nelson, and C. S. Chen, Proc. Natl. Acad. Sci. USA 99, 1758 (2002)

6. L. Tan, Y. P. Kong, L. R. Bao, X. D. Huang, L. J. Guo, S. W. Pang, and A. F. Yee, J. Vac. Sci. Technol. B 21, 2742 (2003)

7. (a) Z.Q. Ouyang, L. Tan, M.Z. Liu, O.S. Judge, X.D. Zhang, H. Li, J. Hu, T.E. Patten, G.Y. Liu, Small, 2, 884-887 (2006); (b) L. Tan, Z.Q. Ouyang, M.Z. Liu, J. Hu, T.E. Patten, G.Y. Liu, J. Phys. Chem. B, 110, 23320(2006)

8. L. Thilly, F. Lecouturier, G. Coffe, and S. Askenazy, IEEE Trans. App. Superconductivity, 12, 1181-1184 (2002) 